\title{
S-1 treatment leading to complete remission of advanced duodenal adenocarcinoma: A case report
}

\author{
LIJUN WANG $^{1 *}$, QUANMAO SONG $^{2 *},{\text { JINPENG } \mathrm{LI}^{3} \text { and XIAOHUA CHEN }}^{2}$ \\ ${ }^{1}$ Department of Computerized Tomography, Shandong Medical Sciences, Jinan, Shandong 250000; \\ ${ }^{2}$ Oncology Department, PKU Care Luzhong Hospital, Zibo, Shandong 255000; ${ }^{3}$ Department \\ of Surgical Oncology (Interventional Therapy), Shandong Cancer Hospital and Institute, \\ Shandong Academy of Medical Sciences, Jinan, Shandong 250117, P.R. China
}

Received April 26, 2015; Accepted July 9, 2015

DOI: $10.3892 / \mathrm{mco} .2015 .607$

\begin{abstract}
Primary duodenal adenocarcinoma (DA) is a rare malignant neoplasm, accounting for $1 \%$ of all gastrointestinal tract carcinomas. This is the case report of a 40 -year-old male patient with a duodenal lesion detected on abdominal magnetic resonance imaging and diagnosed by endoscopy and biopsy as DA. Following surgical resection and histopathological examination, the tumor was confirmed as differentiated duodenal neuroendocrine carcinoma with liver metastasis (TxNxM1). The patient received 8 cycles of palliative chemotherapy with oxaliplatin and S-1 and achieved a clinically complete response, with a treatment-related toxicity profile that was considered as tolerable. Therefore, this regimen exhibited favorable efficacy and a tolerable toxicity profile for the treatment of DA in this case.
\end{abstract}

\section{Introduction}

Duodenal adenocarcinoma (DA) accounts for approximately one-third of all small intestinal malignancies, with the other major tumor types being neuroendocrine carcinoma, sarcoma and lymphoma (1). Due to the relative rarity of small bowel adenocarcinomas (SBAs), the prospective trials limited to this disease are sparse and the optimal therapy for advanced SBA as well as resected node-positive SBA has not been determined (2). Patients with DA exhibit poorer outcomes compared with patients with tumors at others primary sites, such as the jejunum and ileum (1). This is the case report of a patient with DA who underwent treatment with oxaliplatin and S-1 (SOX) and a review of the relevant literature.

Correspondence to: Dr Xiaohua Chen, Oncology Department, PKU Care Luzhong Hospital, 65 Taigong Road, Zibo, Shandong 255000, P.R. China

E-mail: 2940893457@qq.com

${ }^{*}$ Contributed equally

Key words: duodenal adenocarcinoma, drug therapy

\section{Case report}

A 40-year-old man presented to the Shandong Cancer Hospital and Institute on January, 2011 with a month-long history of abdominal pain, repeated vomiting and weight loss. On January 18, 2011, abdominal magnetic resonance imaging was performed, revealing a tumor of the descending duodenum, involving the uncinate process of the pancreas. A deep ulcer $(3.7 \times 4.2 \mathrm{~cm})$, with irregular thickening of the wall of the descending duodenum was confirmed by endoscopy. The histological examination revealed a differentiated duodenal adenocarcinoma (DA). On January 28, 2011, the patient underwent Whipple pancreatoduodenectomy, during which a 2-cm wide tumor was identified in the descending duodenum. The histopathological findings confirmed a 'differentiated adenocarcinoma extending through the duodenal serosa and infiltrating the pancreatic parenchyma without lymph node metastases $(0 \mathrm{~N}+/ 18 \mathrm{~N})$ '. The postoperative period was uneventful, without complications. Two cycles of adjuvant chemotherapy (oral capecitabine monotherapy) were administered following surgery. The clinical and instrumental follow-up revealed disease recurrence: On February 20, 2012, computed tomography (CT) revealed a mass in the right lobe of the liver, which was suspected to be a metastasis. The patient underwent transcatheter arterial chemoembolization on February 28 and radiofrequency ablation on March 5, 2012. At the 2-month follow-up, a CT examination revealed that the right lobe mass had enlarged, with new multiple lymph nodes in the hilar region (Fig. 1) and the disease was considered as progressive. The Eastern Cooperative Oncology Group performance status of the patient was 1. Between May and July, 2012, 4 cycles of palliative chemotherapy were performed, comprising oxaliplatin $(200 \mathrm{mg})$ on day 1 and $\mathrm{S}-1$ (40 $\mathrm{mg} / \mathrm{m}^{2}$ body surface area) administered orally, twice daily on days $1-14$. The cycle was repeated every 3 weeks. In August 20, 2012, an abdominal CT revealed that the right lobe lesions had shrunk and the hilar lymph nodes had disappeared. The patient was classified as being in partial remission (Fig. 2). From August 21, 2012 onwards, 6 cycles of palliative chemotherapy were performed, comprising S-1 (40 mg/m² body surface area) administered orally, twice daily on days $1-28$. The cycle was repeated every 6 weeks. In October 13, 2012, an abdominal CT revealed that 

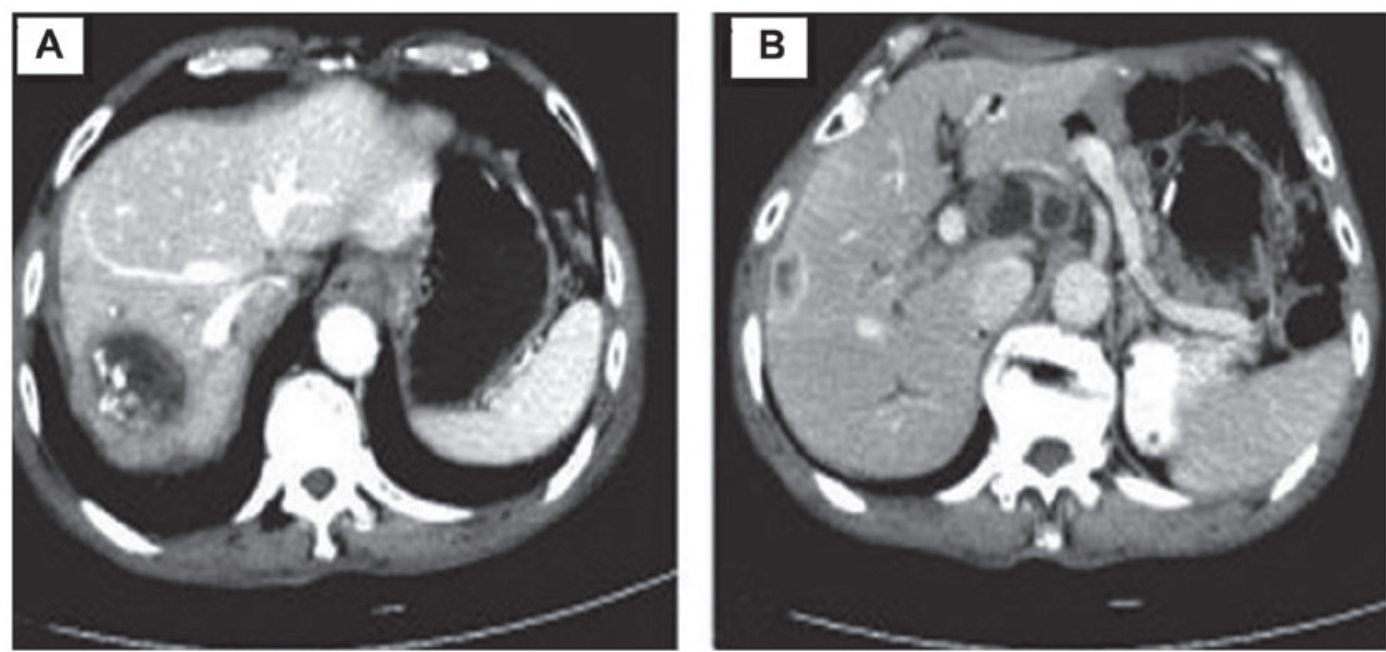

Figure 1. (A) Right hepatic lobe mass due to liver metastasis of duodenal adenocarcinoma and (B) multiple lymph nodes in the hilar region.
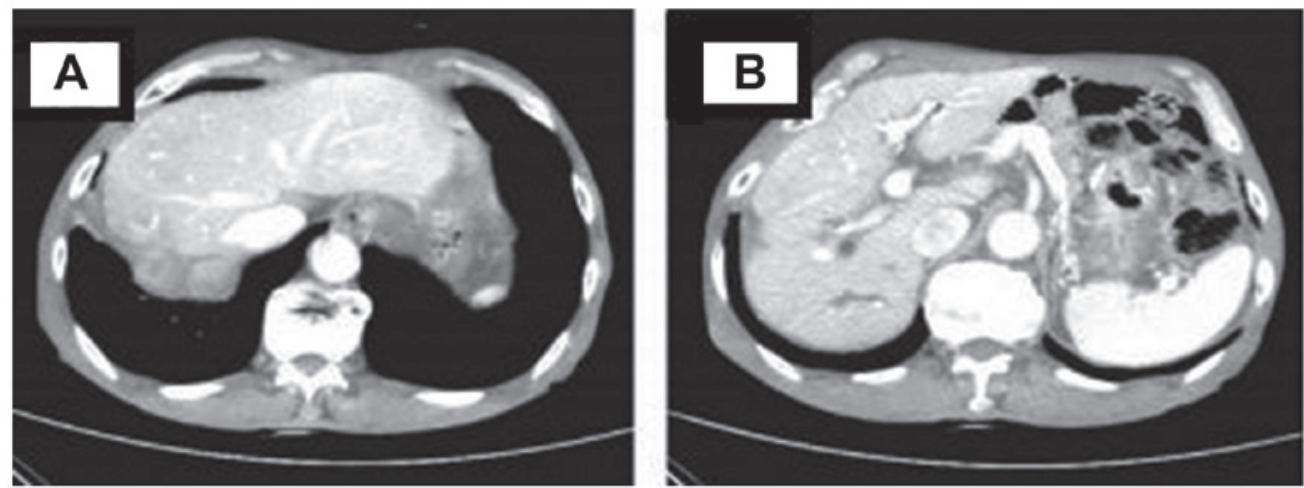

Figure 2. (A) Shrinking of right lobe lesions and (B) disappearance of hilar lymph nodes.
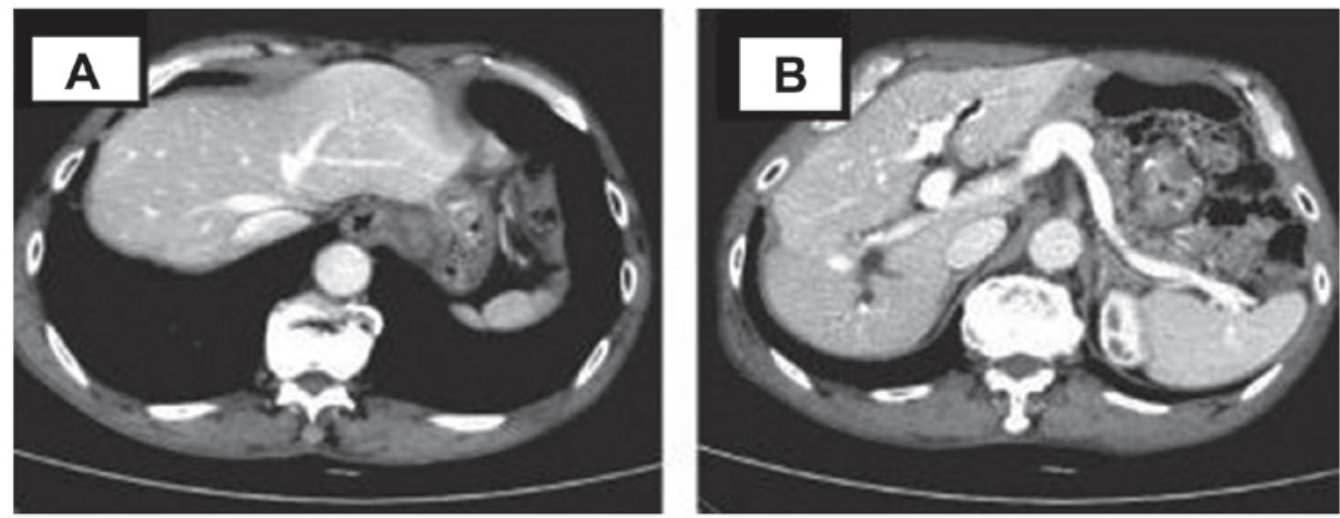

Figure 3. Disappearance of (A) right lobe metastasis and (B) hilar lymph nodes.

the right lobe lesions had disappeared and the patient was in complete remission (CR) (Fig. 3). The primary lesion was not identified at the last follow-up in May, 2013.

According the National Cancer Institute Common Toxicity Criteria, version 3.0 toxicity scale, the most common adverse reactions, including skin pigmentation, leukopenia and diarrhea, subsided 3-4 weeks after treatment completion, spontaneously or with symptomatic treatment. The toxicity was considered tolerable by the patient $(3,4)$.

\section{Discussion}

Although the duodenum is the most common location of small intestinal adenocarcinoma, DA is a rare malignancy, comprising $1 \%$ of all gastrointestinal cancers $(5,6)$. DA is usually diagnosed at an advanced stage and the resectability is low. Even with optimal resection, however, survival is poor and recurrence is common, with a median duration of survival for metastatic disease of $<8$ months $(7,8)$. Ryder et al (9) 
reported on their 40-year experience with DA at the UCLA Medical Center and demonstrated that DAs are characterized by large size, moderate to poor differentiation and invasion of the surrounding fat or mesentery, which are associated with decreased survival. Metastatic lymph node involvement and the location of the tumor within the duodenum were not found to be associated with survival.

Due to the relative rarity of DA, the prospective trials limited to advanced DA treated with chemotherapy are sparse (2). Retrospective studies indicate that chemotherapy may improve the survival of patients with metastatic SBA compared to no treatment (9). Czaykowski and Hui (10) published a study on patients receiving palliative chemotherapy and reported an increase in median survival of $\sim 8$ months compared with patients with advanced disease receiving no chemotherapy. Overman et al (11) conducted a phase II study of capecitabine and oxaliplatin for advanced adenocarcinoma of the small bowel and the ampulla of Vater and reported that the confirmed overall response rate was $50 \%$, the median time to progression was 11.3 months and the median overall survival was 20.4 months.

$\mathrm{S}-1$ is an orally active derivative of 5-fluorouracil (5-FU), which is a fourth-generation oral fluoropyrimidine (12) and has been used instead of 5-FU in certain clinical trials $(13,14)$. S-1 was first approved for the treatment of advanced or metastatic gastric cancer, and there are currently no reports on the treatment of DA. Clinical studies have reported that S-1 in combination with oxaliplatin may achieve a high response rate, ranging between 53 and $59 \%$, with an excellent toxicity profile in the treatment of advanced gastric cancer (15). In this study, the DA patient underwent 4 cycles of the SOX regimen (S-1 $40 \mathrm{mg} / \mathrm{m}^{2} /$ day administered orally, twice daily, with a schedule of 14 days on and 7 days off, and oxaliplatin $130 \mathrm{mg} / \mathrm{m}^{2}$ administered on day 1 ), followed by 6 cycles of single-agent S-1 maintenance chemotherapy, with disappearance of the lesions on imaging and complete remission. The progression-free survival was 14 months. This treatment was associated with high efficacy and an acceptable toxicity profile. In conclusion, the study demonstrated that S-1 may exhibit a favorable efficacy and safety profile in patients with advanced DA, although it requires further validation in clinical trials.

\section{References}

1. Bilimoria KY, Bentrem DJ, Wayne JD, Ko CY, Bennett CL and Talamonti MS: Small bowel cancer in the United States: Changes in epidemiology, treatment, and survival over the last 20 years. Ann Surg 249: 63-71, 2009.
2. Halfdanarson TR, McWilliams RR, Donohue JH and Quevedo JF: A single-institution experience with 491 cases of small bowel adenocarcinoma. Am J Surg 199: 797-803, 2010.

3. Overman MJ, Hu CY, Wolff RA and Chang GJ: Prognostic value of lymph node evaluation in small bowel adenocarcinoma: Analysis of the surveillance, epidemiology, and end results database. Cancer 116: 5374-5382, 2010.

4. Choo R, Pearse M, Danjoux C, et al: Analysis of gastrointestinal and genitourinary morbidity of postoperative radiotherapy for pathologic T3 disease or positive surgical margins after radical prostatectomy using national cancer institute expanded common toxicity criteria.Int J Radiat Oncol Biol Phys 72: 989-995,2008.

5. Kautio AL, Haanpää M, Kautiainen $\mathrm{H}$, et al: Oxaliplatin scale and National Cancer Institute-Common Toxicity Criteria in the assessment of chemotherapy-induced peripheral neuropathy. Anticancer Res 31: 3493-3496, 2011.

6. Overman MJ, Hu C-Y, Kopetz S, Abbruzzese JL, Wolff RA and Chang GJ: A population-based comparison of adenocarcinoma of the large and small intestine: Insights into a rare disease. Ann Surg Oncol 19: 1439-1445, 2012.

7. Gibson MK, Holcroft CA, Kvols LK and Haller D: Phase II study of 5-fluorouracil, doxorubicin, and mitomycin $\mathrm{C}$ for metastatic small bowel adenocarcinoma. Oncologist 10: 132-137, 2005.

8. Lu Y, Fröbom R and Lagergren J: Incidence patterns of small bowel cancer in a population-based study in Sweden: Increase in duodenal adenocarcinoma. Cancer Epidemiol 36: e158-e163, 2012.

9. Ryder NM, Ko CY, Hines OJ, Gloor B and Reber HA: Primary duodenal adenocarcinoma: A 40-year experience. Arch Surg 135: 1070-1075, 2000.

10. Czaykowski P and Hui D: Chemotherapy in small bowel adenocarcinoma: 10-year experience of the British Columbia Cancer Agency. Clin Oncol (R Coll Radiol) 19: 143-149, 2007.

11. Overman MJ, Varadhachary GR, Kopetz S, Adinin R, Lin E, Morris JS, Eng C, Abbruzzese JL and Wolff RA: Phase II study of capecitabine and oxaliplatin for advanced adenocarcinoma of the small bowel and ampulla of Vater. J Clin Oncol 27: 2598-2603, 2009.

12. Wang X, Wang ML, Zhou LY, Lu XY, Yang JF and Yu HG: Randomized phase II study comparing paclitaxel with S-1 vs. S-1 as first-line treatment in patients with advanced gastric cancer. Clin Transl Oncol 15: 836-842, 2013.

13. Ajani JA, Rodriguez W, Bodoky G, Moiseyenko V, Lichinitser M, Gorbunova V, Vynnychenko I, Garin A, Lang I and Falcon S: Multicenter phase III comparison of cisplatin/S-1 with cisplatin/infusional fluorouracil in advanced gastric or gastroesophageal adenocarcinoma study: The FLAGS trial. J Clin Oncol 28: 1547-1553, 2010.

14. Koizumi W, Narahara H, Hara T, Takagane A, Akiya T, Takagi M, Miyashita K, Nishizaki T, Kobayashi O, Takiyama W, et al : S-1 plus cisplatin versus $\mathrm{S}-1$ alone for first-line treatment of advanced gastric cancer (SPIRITS trial): A phase III trial. Lancet Oncol 9: 215-221, 2008.

15. Rosati G, Ferrara D and Manzione L: New perspectives in the treatment of advanced or metastatic gastric cancer. World J Gastroenterol 15: 2689-2692, 2009. 